\title{
The Figure Eight Knot Group is Conjugacy Separable
}

\author{
S. C. Chagas, P. A. Zalesskii *
}

November 4, 2018

\begin{abstract}
We prove that torsion free subgroups of $P G L_{2}(\mathbb{C})$ (abstractly) commensurable with the Euclidean Bianchi groups are conjugacy separable. As a consequence we deduce the result stated in the title.
\end{abstract}

\section{Introduction}

A group $G$ is conjugacy separable if whenever $x$ and $y$ are non-conjugate elements of $G$, there exists some finite quotient of $G$ in which the images of $x$ and $y$ are non-conjugate. The notion of the conjugacy separability owes its importance to the fact, first pointed out by Mal'cev [M-58], that the conjugacy problem has a positive solution in finitely presented conjugacy separable groups. It is known that free products of conjugacy separable groups are again conjugacy separable (see [ [R-71]). The property is not preserved in general by the formation of free products with amalgamation and $H N N$-extensions. The most general result on conjugacy separability of free products with cyclic amalgamation was proved in [R-S-Z-98]. To investigate the property for a non-cyclic amalgamation is much more difficult and only few results exist on the subject (see [R-T-V-95] and [Z-T-95]). One of the most interesting examples of free amalgamated products are Bianchi groups, i.e., the groups $P S L_{2}\left(O_{d}\right)$, where $O_{d}$ denotes the ring of integers of the field $\mathbb{Q}(\sqrt{-d})$ for each square-free positive integer $d$. These groups have long been of interest, not only because of their intrinsic interest as abstract

\footnotetext{
${ }^{*}$ Both authors were supported by CNPq and Capes.
} 
groups, but also because they arise naturally in number theory and geometry. In [W-Z-98] conjugacy separability of Bianchi groups $P S L_{2}\left(O_{d}\right)$ for $d=1,2,7,11$ was proved and conjectured for the rest of them.

In this article we prove the conjugacy separability for torsion free subgroups of $G L_{2}(\mathbb{C})$ (abstractly) commensurable with the Euclidean Bianchi groups, i.e., groups $P S L_{2}\left(O_{d}\right), d=1,2,3,7,11$. We observe that it is not known whether a group commensurable with a conjugacy separable group $G$ (i.e., having a subgroup of finite index isomorphic with some subgroup of finite index of $G$ ) is conjugacy separable.

As a consequence of our main result we deduce that the figure eight knot group is conjugacy separable. Conjugacy separability for knot groups is not known in general, however is known for the trefoiled knot group since it is a free product of cyclic groups with cyclic amalgamation.

From a group theoretic point of view conjugacy separability of a group indicates that it has many subgroups of finite index. The other property that indicates this is subgroup separability. Recall that a group is called subgroup separable (or LERF) if its finitely generated subgroups are closed in the profinite topology. It follows from the definition that commensurability preserves subgroup separability. Although conjugacy separability and subgroup separability do not imply each other, quite often they hold together. The subgroup separability of Bianchi groups was proved recently (see [L-R-2004, Theorem 3.6.1]). Combining this with the results of the paper we deduce that a torsion-free group commensurable with any finitely generated subgroup of an Euclidean Bianchi group is conjugacy separable.

The methods used in the paper are based on Bass-Serre theory of groups acting on trees and its profinite version. The basic notions of these theories are freely used here and can be found in [S-77] and [Z-M-89], [Z-89], [Z-M-90] respectively.

For a group $G$ and elements $g, h \in G$ we shall frequently use notation $g^{h}=h^{-1} g h$ in the paper.

\section{Preliminary results}

\subsection{Centralizers}

Lemma 2.1. If $g \in G=G L_{2}(\mathbb{C}), S L_{2}(\mathbb{C})$ such that $g \notin Z(G)$, then $C=C_{G}(g)$ is abelian.

Proof. Let $g=\left(\begin{array}{ll}a & b \\ c & d\end{array}\right) \in G L_{2}(\mathbb{C}), a d-b c \neq 0$. Using the Jordan 
canonical form we may assume that $g=\left(\begin{array}{ll}a & b \\ 0 & d\end{array}\right), a d \neq 0$. To calculate the centralizer of $g$, we have two cases to consider.

Case 1. The eigenvalues of $g$ are distinct. Hence $g$ is diagonal and so $g=$ $\left(\begin{array}{ll}a & 0 \\ 0 & d\end{array}\right)$. Then a straightforward calculation shows that the centralizer of $g$ is equal to

$$
C_{G L_{2}(\mathbb{C})}(g)=\left\{\left(\begin{array}{cc}
x & 0 \\
0 & w
\end{array}\right) \mid x w \neq 0\right\}
$$

Case 2. If $a=d$, i.e., the eigenvalues are equal, a straightforward calculation shows that the centralizer of $g$ is

$$
C_{G L_{2}(\mathbb{C})}=\left\{\left(\begin{array}{ll}
x & y \\
0 & x
\end{array}\right) \mid x \neq 0\right\} .
$$

Thus in both cases the centralizer of a non-central element $g \in G L_{2}(\mathbb{C})$ is abelian.

Finally observe that $C_{G L_{2}(\mathbb{C})}(g) \cap S L_{2}(\mathbb{C})=C_{S L_{2}(\mathbb{C})}(g)$ and therefore the centralizer of a non-central element of $g \in S L_{2}(\mathbb{C})$ is also abelian.

Lemma 2.2. Let $\bar{g} \in P G L_{2}(\mathbb{C})$ be an element of infinite order. Then the centralizer $C_{P G L_{2}(\mathbb{C})}(\bar{g})$ of $\bar{g}$ is abelian. Moreover, $C_{\Gamma_{d}}(\bar{g})$ is virtually free abelian of rank at most 2 , where $\Gamma_{d}=P S L_{2}\left(O_{d}\right)$.

Proof. Consider the projection $\varphi: G L_{2}(\mathbb{C}) \longrightarrow P G L_{2}(\mathbb{C})=G L_{2}(\mathbb{C}) / Z$, where $Z=Z\left(G L_{2}(\mathbb{C})\right)=\left\{\left(\begin{array}{ll}a & 0 \\ 0 & a\end{array}\right) \mid a \in \mathbb{C}\right\}$ is the center of $G L_{2}(\mathbb{C})$. We use $\bar{g}$ for the image of $g$ in $G L_{2}(\mathbb{C}) / Z$. Pick $1 \neq \bar{g} \in P G L_{2}(\mathbb{C})$.

We write down the inverse image of the centralizer of the element $\bar{g} \in$ $P G L_{2}(\mathbb{C})$ :

$$
\varphi^{-1}\left(C_{P G L_{2}(\mathbb{C})}(\bar{g})\right)=\left\{h \in G L_{2}(\mathbb{C}) \mid h g h^{-1}=g z, \text { for some } z \in Z\right\} .
$$

Using the Jordan canonical form we may suppose that $g=\left(\begin{array}{cc}x & y \\ 0 & w\end{array}\right)$ and $g z=\left(\begin{array}{cc}a x & a y \\ 0 & a w\end{array}\right)$. Since conjugate matrices have the same eigenvalues, theses matrices can be conjugate in $G L_{2}(\mathbb{C})$ only if either $a=1$ or $a w=$ $x$ and $w=a x$. In the second case we have $a^{2}=1$ and so $a=-1$. 
But the matrix $g=\left(\begin{array}{cc}x & y \\ 0 & -x\end{array}\right)$ has finite order in $P G L_{2}(\mathbb{C})$, because $g^{2}=\left(\begin{array}{cc}x^{2} & 0 \\ 0 & x^{2}\end{array}\right)$ is in the center of $G L_{2}(\mathbb{C})$. Since $g$ has infinite order by hypothesis, $a=1$.

Thus we have

$$
\varphi^{-1}\left(C_{P G L_{2}(\mathbb{C})}(\bar{g})\right)=C_{G L_{2}(\mathbb{C})}(g) .
$$

Since $Z \leq C_{G L_{2}(\mathbb{C})}(g)$, we have that $C_{P G L_{2}(\mathbb{C})}(g)$ is the quotient group of the centralizer of $g$ in $G L_{2}(\mathbb{C})$ modulo the center, i.e., $C_{P G L_{2}(\mathbb{C})}(g) \cong$ $C_{G L_{2}(\mathbb{C})}(g) / Z$. By Lemma 2.1 the centralizer of $g$ in $P G L_{2}(\mathbb{C})$ is abelian.

The second part of the lemma follows from the fact that the torsion free abelian subgroups in $\Gamma_{d}$ are free abelian of rank at most 2, see Fine [F-89] in page 107 .

Corollary 2.3. A subgroup $G$ of $P G L_{2}(\mathbb{C})$ does not contain the generalized dihedral group. In particular, this is so for Bianchi groups.

Proof. If the generalized dihedral group $E \cong\left\langle x, y \mid x^{2}=y^{2}\right\rangle$ is a subgroup of $P G L_{2}(\mathbb{C})$ then $E \leq C_{G}\left(x^{2}\right)$ which is an abelian group by Lemma 2.1

\subsection{The Profinite topology}

The profinite topology on a group $G$ is the topology where the collection of all finite index normal subgroups of $G$ serves as a fundamental system of neighborhoods of the identity element $1 \in G$, turning $G$ into a topological group. The completion $\widehat{G}$ of $G$ with respect to this topology is called the profinite completion of $G$ and can be expressed as an inverse limit

$$
\widehat{G}=\lim _{N \in \aleph} G / N
$$

where $\mathcal{N}=\left\{N \mid N \unlhd_{f} G\right\}$. Thus $\widehat{G}$ becomes a profinite group, i.e. a compact totally disconnected topological group. Moreover, there exists a natural homomorphism $\iota: G \longrightarrow \widehat{G}$ that sends $g \mapsto(g N)$, this homomorphism is a monomorphism when $G$ is residually finite. If $S$ is a subset of a topological group $\widehat{G}$, we denote by $\bar{S}$ its closure in $\widehat{G}$. The profinite topology on $G$ is induced by the topology of $\widehat{G}$. Note that for a subgroup $H$ of $G$, the profinite topology of $H$ can be stronger than the topology induced by the profinite topology of $G$.

Next proposition expresses the conjugacy separability property of $G$ in terms of the profinite topology and we shall use it freely in the paper. 
Proposition 2.4. Let $G$ be a group, then the following conditions are equivalent:

(i) $G$ is conjugacy separable;

(ii) for each $x \in G$, the conjugacy class $x^{G}$ of $x$ is closed in the profinite topology. In particular $G$ is residually finite;

(iii) $G$ is residually finite and for each pair of elements $x, y \in G$ such that $y=x^{\gamma}$, for some $\gamma \in \widehat{G}$, there exists $g \in G$ such that $y=x^{g}$.

Lemma 2.5. Let $G$ be a residually finite group, $U$ a finite index subgroup of $G$ and $H$ a subgroup of $G$. Then $\bar{U} \cap \bar{H}=\overline{U \cap H}$.

Proof. We just need to proof that $\bar{U} \cap \bar{H} \leq \overline{U \cap H}$, since clearly $\overline{U \cap H} \leq$ $\bar{U} \cap \bar{H}$.

Consider the following commutative diagram

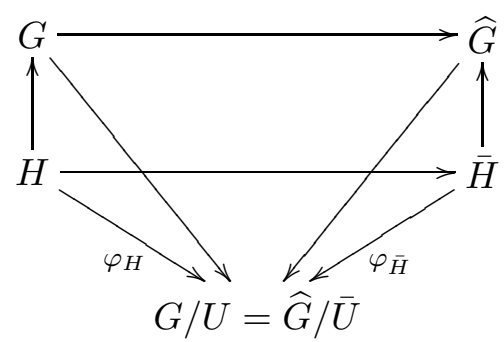

Note that $\operatorname{Im} \varphi_{\bar{H}}=\operatorname{Im} \varphi_{H}, \operatorname{Ker}\left(\varphi_{\bar{H}}\right)=\bar{H} \cap \bar{U}$ and $\operatorname{Ker}\left(\varphi_{H}\right)=H \cap U$. Therefore, $[\bar{H}:(\bar{H} \cap \bar{U})]=\left|\operatorname{Im} \varphi_{\bar{H}}\right|=\left|\operatorname{Im} \varphi_{H}\right|=[H:(H \cap U)] \geq[\bar{H}:$ $\overline{H \cap U}$. It follows that $\bar{U} \cap \bar{H}=\overline{U \cap H}$ as needed.

Let $G=\pi_{1}(\mathcal{G}, \Gamma)$ be a fundamental group of the graph of conjugacy separable groups $(\mathcal{G}, \Gamma)$. Then the profinite topology on $G$ might induce a weaker topology on the vertex and edge groups than their own profinite topologies. This prevents to use effectively the conjugacy separability of the vertex and edge groups for proving the conjugacy separability of $G$. Thus it is natural to assume that the profinite topology on $G$ is reasonably "strong". We formalize it in the following definition.

Definition 2.6. Let $G$ be a group acting on a tree $S$ such that $S / G$ is finite. We say that the profinite topology on $G$ is efficient, if $G$ is residually finite, the edge stabilizers $G_{e}$ and the vertex stabilizers $G_{v}$ are closed in the profinite topology on $G$ and the profinite topology of $G$ induces the full profinite topology on the vertex and edge stabilizers. 
If $S / G$ is finite, then we can define a profinite graph

$$
\widehat{S}=\lim _{U \triangleleft_{f} G} S / U
$$

where $U$ ranges over the normal subgroups of finite index of $G$. The profinite completion $\widehat{G}$ acts continuously on $\widehat{S}$ and $\widehat{S} / \widehat{G}=S / G$. Moreover, $\widehat{S}$ is a profinite tree (see Proposition 3.8 in [Z-M-89]).

Remark 2.7. Suppose the profinite topology on $G$ is efficient. Then $S$ embeds naturally in $\widehat{S}$. This follows from the fact that $G / G_{m}$ embeds in $\widehat{G} / \widehat{G}_{m}$ because $G_{m}$ are closed in $G$ for all $m \in S$. Moreover, $S$ is dense in $\widehat{S}$.

We define the standard trees $S(G)$ on which $G$ acts (respectively, $S(\widehat{G})$ on which $\widehat{G}$ acts) only for the cases of an amalgamated free product $G=$ $G_{1} *_{H} G_{2}$ (respectively, $\widehat{G}=\widehat{G}_{1} \amalg_{\widehat{H}} \widehat{G_{2}}$ ) and an HNN-extension $G=$ $\operatorname{HNN}\left(G_{1}, H, t\right)$ (respectively, $\widehat{G}=H N N\left(\widehat{G}_{1}, \widehat{H}, t\right)$ ) since we shall use them only for these cases.

- Let $G=G_{1} *_{H} G_{2}$. Then the vertex set is $V(S(G))=G / G_{1} \cup G / G_{2}$, the edge set is $E(S(G))=G / H$, and the initial and terminal vertices of an edge $g H$ are respectively $g G_{1}$ and $g G_{2}$.

- Similarly, let $\widehat{G}=\widehat{G}_{1} \amalg_{\widehat{H}} \widehat{G_{2}}$. Then the vertex set is $V(S(\widehat{G}))=$ $\widehat{G} / \widehat{G}_{1} \cup \widehat{G} / \widehat{G}_{2}$, the edge set is $E(S(\widehat{G}))=\widehat{G} / \widehat{H}$, and the initial and terminal vertices of an edge $g \widehat{H}$ are respectively $g \widehat{G}_{1}$ and $g \widehat{G}_{2}$.

- Let $G=H N N\left(G_{1}, H, t\right)$. Then the vertex set is $V(S(G))=G / G_{1}$, the edge set is $E(S(G))=G / H$, and the initial and terminal vertices of an edge $g H$ are respectively $g G_{1}$ and $g t G_{1}$.

- Similarly Let $\widehat{G}=H N N\left(\widehat{G}_{1}, \widehat{H}, t\right)$. Then the vertex set is $V(S(\widehat{G}))=$ $\widehat{G} / \widehat{G}_{1}$, the edge set is $E(S(\widehat{G}))=\widehat{G} / \widehat{H}$, and the initial and terminal vertices of an edge $g \widehat{H}$ are respectively $g \widehat{G}_{1}$ and $g t \widehat{G}_{1}$.

It follows that $S(\widehat{G})=\widehat{S(G)}$ and so Remark 2.7 applies for $S(G)$.

Lemma 2.8. Let $G=G_{1} \coprod G_{2} \amalg \cdots \amalg G_{k}$ be a free profinite product of profinite groups. Then $G_{i}$ are isolated in $G$, i.e., if $g^{n} \in G_{i}$, for some natural number $n$ and some $g \in G$ of infinite order, then $g \in G_{i}$.

Proof. By an obvious induction on the number of free factors it suffices to consider the case $k=2$. Let $g \in G$ be an element such that $g^{n} \in$ $G_{i}$, we show that $g \in G_{i}$, for some $i=1,2$. Consider the action of $G$ on the standard profinite tree $S(G)$ (one can use its construction above for 
amalgamated free product and take an amalgamating subgroup to be trivial). Then $g^{n}$ fixes a vertex in $S(G)$. Hence $\langle g\rangle /\left\langle g^{n}\right\rangle$ is a finite group acting on the subtree of fixed points $T^{g^{n}}$ (see Theorem 2.8 in [Z-M-89]). By Theorem 2.10 in [Z-M-89] $\langle g\rangle /\left\langle g^{n}\right\rangle$ fixes a vertex in $T^{g^{n}}$ and so $g$ fixes the same vertex, say $h G_{j}$. It follows that $h g h^{-1} \in G_{j}$. Since $G_{i} \cap h G_{j} h^{-1}=1$ for $i \neq j$ as one can easily see mapping $G$ onto $G_{1} \times G_{2}$ we deduce that $i=j$. Since $G_{i} \cap G_{i}^{x}=1$ for $x \in G \backslash G_{i}$ (see Theorem 9.1.12 in [RZ]) $g \in G_{i}$, as needed.

We distinguish two types of elements of a group $G$ acting on a tree $S$ : we say that a non-trivial element $g$ is hyperbolic if it does not stabilize any vertex of $S$. Otherwise, $g$ is called non-hyperbolic. The following proposition will be used in some of our proofs.

Proposition 2.9 (Proposition 24 in [S-77]). Let $G$ be an amalgamated free product or an $H N N$ - extension and $S=S(G)$ its standard tree. Suppose that an element $a \in G$ is hyperbolic. Put $m=\min _{v \in V(S)} l[v, a v]$ and $T_{a}=\{v \in V(S) \mid l[v, a v]=m\}$, where $l[v, a v]$ represents the length of the geodesic $[v, a v]$. Then $T_{a}$ is the vertex set of a straight line (that is, a doubly infinite chain of $S(G)$ ), on which a acts as a translation of amplitude $m$. Moreover, all $\langle a\rangle$-invariant subtrees of $S(G)$ contain $T_{a}$. Finally if $v \in T_{a}$, then $T_{a}=\langle a\rangle[v, a v[$.

The followings proposition gives information on the closure of a straight line in $S(\widehat{G})$, and shows that a hyperbolic element of $G$ is hyperbolic in the profinite sense.

Proposition 2.10. Let $G$ be an amalgamated free product or an $H N N$ extension and $S=S(G)$ its standard tree. Suppose also that the profinite topology on $G$ is efficient. Let $a \in G$ be a hyperbolic element and $T_{a}$ the corresponding straight line. Then:

(i) $\overline{\langle a\rangle}$ acts freely on the tree $S(\widehat{G})$;

(ii) $\overline{T_{a}}=\overline{\langle a\rangle}\left[v\right.$, av [, where $v$ is a vertex of $T_{a}$;

(iii) $\overline{\langle a\rangle}$ acts freely on the profinite tree $\overline{T_{a}}$, and $\overline{\langle a\rangle} \cong \widehat{\mathbb{Z}}$;

(iv) $T_{a}$ is a connected component of $\overline{T_{a}}$ considered as an abstract graph, in others words, the only vertices of $\overline{T_{a}}$ that are at a finite distance from a vertex of $T_{a}$ are those of $T_{a}$.

Proof. Item (i) is proved in Proposition 2.9 [R-Z-96]. Items (ii) and (iii) are proved in Lemma 4.1 [R-S-Z-98]. 
To prove (iv) we use the same argument as in Lema 4.3 [R-S-Z-98]. Let $w$ be a vertex of $\overline{T_{a}}$ that is at finite distance from $v$. We show that $w \in T_{a}$. Suppose that $w \notin T_{a}$, then there exists an edge $e^{\prime}$ of the geodesic $[v, w]$ that is not in $T_{a}$ and we can assume that the initial vertex of $e^{\prime}$ is $v$. Since $\overline{T_{a}}=\overline{\langle a\rangle}\left[v, a v\left[\right.\right.$, there exists $\alpha \in \overline{\langle a\rangle}$ such that $\alpha e=e^{\prime}$, where $e$ is an edge of $\left[v, a v\left[\right.\right.$. Let $w_{0}$ be the origin of the edge $e$, then $\alpha w_{0}=v$. Thus $\alpha w_{0}=v \in T_{a}$, and since $T_{a}=\langle a\rangle[v, a v[$ there exists $\beta \in\langle a\rangle$ such that $\beta \alpha w_{0} \in\left[v, a v\left[\right.\right.$, so $\beta \alpha=1$, i.e. $\alpha \in\langle a\rangle$. Then $\alpha e=e^{\prime} \in T_{a}$ and so $w \in T_{a}$, a contradiction.

We shall need the following generalization of the M. Hall Theorem to free products. Remind that a group $G$ is called LERF if each finitely generated subgroup is closed in the profinite topology of $G$.

Proposition 2.11 ([G-M-S-2003]). Let $G_{1}, \ldots, G_{m}$ be LERF groups and let $H$ be a finitely generated subgroup of the free product $G=G_{1} * \cdots *$ $G_{m}$. Then there exists a subgroup $U$ of $G$ of finite index and (Kurosh-type) decompositions

$$
U=U_{1} * \cdots * U_{t} \text { and } H=H_{1} * \cdots * H_{t}
$$

such that

(a) $H_{i} \leq U_{i}, i=1, \ldots, t$;

(b) For each $i=1, \ldots, t-1, U_{i}$ is a subgroup of finite index of a conjugate of some $G_{j}, j=1, \ldots, m$, i.e., $U_{i}=\tau G_{j} \tau^{-1}$ for some $\tau \in G$;

(c) $U_{t}$ is a free group of finite rank and $H_{t}$ is a free factor of $U_{t}$.

Sometimes we need to separate the conjugacy class of a subset or a subgroup of $G$ in a finite quotient rather than the conjugacy class of an element. The following definition formalizes this.

Definition 2.12. A subgroup $H$ of a group $G$ is called conjugacy distinguished, if $\cup_{g \in G} H^{g}$ is closed in the profinite topology of $G$. Or equivalently, for every element $g \in G$ such that $g^{\gamma} \in \bar{H}$, where $\gamma \in \widehat{G}$, there exists $\delta \in G$ such that $g^{\delta} \in H$.

The following proposition collects important results about free product of virtually abelian and virtually free groups that will be used in the paper.

Proposition 2.13. Let $G$ be a finitely generated free product of virtually abelian groups and a virtually free groups, then:

1. G is conjugacy separable; 


\section{G is LERF;}

3. The profinite topology on $G$ induces the (full) profinite topology on every finitely generated subgroup of $G$;

4. For each pair of finitely generated subgroups $H_{1}, H_{2}$ of $G$, the product $H_{1} H_{2}$ is a closed subset of $G$, i.e., $\overline{H_{1} H_{2}} \cap G=H_{1} H_{2}$, where $\overline{H_{1} H_{2}}$ is the closure of $H_{1} H_{2}$ in $\widehat{G}$;

5. Every finitely generated subgroup $H$ of $G$ is conjugacy distinguished;

Proof. Item 1 follows from the fact that a free product of conjugacy separable groups is conjugacy separable and that virtually abelian and virtually free groups are conjugacy separable (see [Dy-79] and [R-71]). Item 2 follows from the fact that a free product of LERF groups is LERF (see [Ro-69] and [B-71]). Item 3 follows from 2 , because every subgroup $H$ of finite index of a finitely generated subgroup $K$ is finitely generated and so is closed in the profinite topology of $G$. Indeed, this means that $H$ is the intersection of subgroups of finite index of $G$ and so for some of them, say $U$ one has $U \cap K=H$. Item 4 was proved in [R-Z-04] and [C-2001].

5. Let $g \in G$ and suppose that $g^{\gamma} \in \bar{H}$, for some $\gamma \in \widehat{G}$. Let $\langle g\rangle$ be the subgroup generated by $g$. Then by Proposition 2.11 there is a subgroup $U$ of finite index such that $\langle g\rangle \leq U \leq G$, and $\langle g\rangle$ is a subgroup of a free factor of the Kurosh decomposition for $U$. Hence $g$ is a non-hyperbolic element of $U$. Since $U \leq_{f} G, \widehat{G}=\widehat{U} G$, so $\gamma=u \sigma$, where $u \in \widehat{U}$ and $\sigma \in G$. Therefore substituting $H$ by $H^{\sigma^{-1}}$ we may assume that $\gamma \in \widehat{U}$.

Now observe that $H \cap U$ is a finitely generated subgroup of $U$, because $H \cap U$ has finite index in $H$. Hence by Proposition 2.11, there exists a finite index subgroup $U_{0}$ of $U$ such that $H \cap U \leq U_{0} \leq U$ and the Kurosh decomposition of $H \cap U$ is compatible with the Kurosh decomposition of $U_{0}$. Namely the decompositions of $U_{0}$ and $H \cap U$, are respectively as follows

$$
\begin{aligned}
U_{0} & =U_{0}^{1} * \ldots * U_{0}^{m} \\
H \cap U & =H_{1} * \ldots * H_{m},
\end{aligned}
$$

where $H_{i} \leq U_{0}^{i}$. Since $U_{0} \leq{ }_{f} U, \widehat{U}=\widehat{U_{0}} U$, so $\gamma=g^{\prime} \delta$, where $g^{\prime} \in \widehat{U_{0}}$ and $\delta \in U$. Thus substituting $U_{0}, H$ and $H \cap U$ (together with their Kurosh decompositions) by conjugates $U_{0}^{\delta-1}, H^{\delta^{-1}}$ and $(H \cap U)^{\delta^{-1}}$ in $G$, we can suppose that $\gamma \in \widehat{U_{0}}$. Since $g^{\gamma} \in \bar{H}$ and $g^{\gamma} \in \bar{U}$, one has $g^{\gamma} \in \bar{H} \cap \bar{U}$.

Since $U_{0} \leq{ }_{f} U$, then $g^{n} \in U_{0}$ for some natural number $n$. Since $g$ is an element of a free factor of $U, g^{n}$ is an element of a conjugate of a free factor of $U_{0}$, so without loss of generality we suppose that $g^{n} \in U_{0}^{1}$. Now

$$
\bar{U}_{0}=\widehat{U}_{0}=\widehat{U}_{0}^{1} \amalg \ldots \amalg \widehat{U}_{0}^{m} .
$$


Then by the projective limit argument one deduces that $g^{n \gamma}$ is conjugate to an element of a free factor of

$$
\overline{H \cap U}=\bar{H}_{1} \amalg \ldots \amalg \bar{H}_{m} .
$$

Indeed, $\bar{U}_{0}=\lim _{\longleftarrow} \bar{U}_{0 V}$, where

$$
\overline{U_{0 V}}=\widehat{U}_{0}^{1} /\left(\widehat{U}_{0}^{1} \cap V\right) \amalg \ldots \amalg \widehat{U}_{0}^{m} /\left(\widehat{U}_{0}^{m} \cap V\right)
$$

and $V$ ranges over all open subgroups of $\bar{U}_{0}$. Then

$$
\overline{\left(H \cap U_{0}\right)_{V}}=\widehat{H}_{0}^{1} /\left(\widehat{H}_{0}^{1} \cap V\right) \amalg \ldots \amalg \widehat{H}_{0}^{m} /\left(\widehat{H}_{0}^{m} \cap V\right) .
$$

Since the image $g_{V}^{n \gamma}$ of $g^{n \gamma}$ in $\overline{\left(H \cap U_{0}\right)_{V}}$ has finite order, by Theorem 1 in [H-R-85] it is conjugate in $\overline{\left(H \cap U_{0}\right)_{V}}$ to an element of a free factor for every $V$. Therefore $g^{n \gamma}$ is conjugate in $\overline{H \cap U}$ to an element of $\bar{H}_{i}$. By Theorem B' in [H-R-85] $\bar{U}_{0}^{1} \cap H_{j}^{u}=1$ for all $u \in \bar{U}_{0}, j>1$. Hence $g^{n \gamma}$ is conjugate to $\bar{H}_{1}$ in $\overline{U_{0} \cap H}$.

Thus we may assume that $g^{n \gamma} \in \bar{H}_{1}$. By Lemma $2.5 \bar{H} \cap \bar{U}_{0}=\overline{H \cap U_{0}}$, therefore, $g^{\gamma} \in \bar{H}_{1}$, since $\bar{H}_{1}$ is isolated in $\overline{H \cap U}=\bar{H}_{1} \amalg \ldots \amalg \bar{H}_{m}$ (see Lemma 2.8). Since $g^{n} \in U_{0}^{1}$ and $g^{n \gamma} \in U_{0}^{1}$, by Theorem 9.1.12 in [R-Z-96] the conjugating element $\gamma \in \bar{U}_{0}^{1}$. It follows that $g \in U_{0}^{1}=G \cap \overline{U_{0}^{1}}$. But $U_{0}^{1}$ is either virtually free or virtually abelian, so $H_{1}$ is conjugacy distinguished in $U_{0}^{1}$. This finishes the proof.

If $\Gamma$ is a profinite graph then its connected component is a maximal connected profinite subgraph of $\Gamma$. Note that it coincide with the closure of a connected component of $\Gamma$ considered as an abstract graph, since the closure of a connected abstract subgraph is a connected profinite subgraph. If a profinite group $G$ acts on $\Gamma$ and $C$ its connected component we denote by $\operatorname{Stab}_{G}(C)$ the maximal subgroup of $G$ leaving $C$ invariant.

Lemma 2.14. Let $G$ be a profinite group acting on a profinite graph $S$ and let $m_{1}, m_{2}$ be elements of a connected component $C$ of $S$. Then

$$
C / \operatorname{Stab}_{G}(C) \subseteq S / G .
$$

Proof. We need to show that if $g \in G$ with $g m_{1}=m_{2}$, then $g$ leaves $C$ invariant, i.e. $g \in \operatorname{Stab}_{G}(C)$. Since $m_{2} \in C \cap g C, C \cup g C$ is connected and so $C=g C$. 
Lemma 2.15. Let $G$ be a group acting on a profinite tree $S$ such that $S / G$ is finite. Let $\Delta$ be a profinite subgraph of $S$ and e be an edge in $S$. Suppose a connected component $C$ of $\Gamma:=\Delta \backslash$ Ge contains at least one edge. Then there exist edges $e^{\prime} \in C$ and $e_{0} \in G e \cap \Delta$ that have a common vertex $v$.

Proof. Consider the profinite graph $\Delta_{\Gamma}$ obtained from $\Delta$ by collapsing all connected components of $\Gamma$. By Proposition 3.2 in [Z-92] $\Delta_{\Gamma}$ is a profinite tree. Let $v_{C}$ be the vertex that is the image of the connected component $C$. Now since $E\left(\Delta_{\Gamma}\right)$ is compact (because $\Delta \backslash \widehat{G} e$ is open and closed), by Proposition 2.15 in [Z-M-89] there exists an edge $\overline{e_{0}} \in \Delta_{\Gamma}$ that has $v_{C}$ as a vertex, say $v_{C}=d_{0}\left(\bar{e}_{0}\right)$. Let $e_{0}=\pi_{\Gamma}^{-1}\left(\overline{e_{0}}\right)$, where $\pi_{\Gamma}: \Delta \longrightarrow \Delta_{\Gamma}$ is a quotient map. Then $d_{0}\left(e_{0}\right) \in C$ and since $E(C)=E(S(G)) \cap C$ is compact, by Proposition 2.15 [Z-M-89] there exist $e^{\prime} \in E(C)$ having $d_{0}\left(e_{0}\right)$ as a vertex. Note also that since $\pi_{\Gamma}(E(\Delta))=\pi_{\Gamma}(\widehat{G} e)$ one has $e_{0} \in \widehat{G} e$.

Lemma 2.16. Let $G$ be a group that acts on a tree $S$ such that $S / G$ is finite and the edge stabilizers are finitely generated. Suppose that the profinite topology on $G$ is efficient and there exists an epimorphism $\tau: G \longrightarrow K$ such that the restriction of $\tau$ to the vertex stabilizer $G_{v}$ is an isomorphism for each $v \in V(S)$. Suppose every finitely generated subgroup of $K$ is conjugacy distinguished. Then the edge stabilizers of $G$ are conjugacy distinguished in $G$.

Proof. Let $e \in S$ be an edge. We have to show that $G_{e}$ is conjugacy distinguished in $G$. Let $g \in G$ and $\gamma \in \widehat{G}$ be such that $g^{\gamma} \in \widehat{G_{e}}$, we need to show that there exists $\gamma_{1} \in G$ such that $g^{\gamma_{1}} \in G_{e}$. Observe that by item (i) of Proposition $2.10 \mathrm{~g}$ is a non-hyperbolic element of $G$, therefore $g$ stabilizes a vertex $v$ in $S$. Fix a connected transversal $T$ of $S / G$ in $S$. Without loss of generally we can suppose that $v$ and $e$ belong to $T$. We use induction on the number of edges of the geodesic $[v, e]$ in $T$.

Suppose $[v, e]$ has one edge only. Since $\tau$ restricted to $G_{v}$ is an isomorphism to $K, \tau\left(G_{e}\right)$ is conjugacy distinguished in $K$ by Proposition 2.13 ( $\tau\left(G_{e}\right)$ is finitely generated). Hence the exists $k \in K$ such that $\tau(g)^{k} \in$ $\tau\left(G_{e}\right)$ and since $\left.\tau\right|_{G_{v}}$ is an isomorphism to $\mathrm{K}$, there exists $\delta \in G_{v}$ such that $\tau(\delta)=k$. Then $\tau(g)^{\tau(\delta)} \in \tau\left(G_{e}\right)$, and consequently $g^{\delta} \in G_{e}$.

Suppose now that $[v, e]$ has more than one edge. Since $g$ fixes $v$ and $\gamma^{-1} e$ by Theorem 2.8 in [Z-M-89] $g$ fixes the geodesic $\left[v, \gamma^{-1} e\right]$, i.e., the minimal profinite subtree which contains $v$ and $\gamma^{-1} e$. Denote by $C$ the connected component of $\left[v, \gamma^{-1} e\right] \backslash \widehat{G} e$ containing $v$. Note that $C$ contains at least one edge since otherwise $v$ would be a vertex of a translation of $e$, contradicting the assumption that $[v, e]$ has more than one edge. Then by 
Lemma 2.15 there are edges $e^{\prime} \in C$ and $e_{0} \in \widehat{G} e \cap\left[v, \gamma^{-1} e\right]$ that have a vertex in common, say $d_{0}\left(e_{0}\right)$.

Let $\nu: S(\widehat{G}) \rightarrow S(\widehat{G}) / \widehat{G}$ be the natural epimorphism. Observe that $\nu(e)=\widehat{G} e$ and $\nu(v) \in(S / G) \backslash \nu(e)$. Let $\widehat{\Pi}$ be the maximal subgroup of $\widehat{G}$ that leaves invariant the connected component $\widehat{C}$ of $S(\widehat{G}) \backslash \widehat{G} e$ containing $v$, i.e. $\widehat{\Pi}=S t a b_{\widehat{G}}(\widehat{C})$. By Lemma $\left.2.14 \nu\right|_{\widehat{C}}$ coincides with the factorization of $\widehat{C}$ modulo $\widehat{\Pi}$. Therefore, replacing $\gamma$ with its multiple $x \gamma$ for some $x \in \widehat{\Pi}$ we may assume that $e^{\prime} \in T$. But $\left[v, e^{\prime}\right]$ has less edges then $[v, e]$ so by the induction hypothesis $g$ is conjugate to an element of $G_{e^{\prime}}$ in $G$. Hence we can assume that $g \in G_{e^{\prime}}$. It follows that $g \in d_{0}\left(e_{0}\right)$ and replacing $v$ by $d_{0}\left(e_{0}\right)$ we may assume that $v$ is the initial vertex of $e_{0}$. But changing $\gamma$ again we may assume that $e_{0}$ is in $T$ (we loosing $e \in T$ of course), so by the induction hypothesis again we deduce that $g$ is conjugate in $G$ to an element of $G_{e_{0}}$. However, we observe now that $T$ contains unique edge of the orbit $\widehat{G} e$ so our new $e_{0} \in T$ is the same as our old $e$. Thus $g$ is conjugate to an element of $G_{e}$ in $G$ as required.

Theorem 2.17. Let $G$ be a torsion free group that does not contain subgroups isomorphic to a generalized dihedral. Let $H$ be a finite index subgroup of $G$ such that:

1. $H$ is conjugacy separable;

2. for each $h \in H, C_{H}(h)$ is a free abelian group of rank at most 2 .

Then $G$ is conjugacy separable.

Proof. Let $g_{1}, g_{2} \in G$ such that $g_{2}=g_{1}^{\gamma}$, for some $\gamma \in \widehat{G}$. Since $H$ has finite index in $G, g_{1}^{m}, g_{2}^{m} \in H$, where $m=|G: H|$.

Observe that $\widehat{G}=G \widehat{H}$, so that we can write $\gamma=d \gamma_{0}$, where $\gamma_{0} \in \widehat{H}$ and $d \in G$. Therefore $g_{2}^{m}=\left(g_{1}^{\gamma}\right)^{m}=\left(g_{1}^{m}\right)^{\gamma}=\left(g_{1}^{m}\right)^{d \gamma_{0}}$. Now substituting $g_{1}$ by $g_{1}^{d}$, we can suppose that $\gamma \in \widehat{H}$. Thus $g_{1}^{m}$ and $g_{2}^{m}$ are conjugated in $\widehat{H}$, and since $H$ is conjugacy separable there exists $h \in H$ such that $g_{1}^{m}=\left(g_{2}^{h}\right)^{m}$. Hence $g_{1}^{m}=\left(g_{2}^{m}\right)^{h}$, so $g_{1}^{m}$ and $g_{2}^{m}$ are conjugate in $H$. Thus we can suppose that $g_{1}^{m}=g_{2}^{m}$. Let $N=\left\langle g_{2}^{m}\right\rangle$ and $K=\left\langle g_{1}, g_{2}\right\rangle$. Then $K$ centralizes $N$. Since $C_{G}(N)$ is a virtually free abelian group of rank at most $2, K$ is a virtually free abelian group of rank at most 2 . If $K$ is virtually cyclic, then since $K$ is torsion free, by Theorem 3.5 in [D-80] $K$ is cyclic and therefore $g_{1}=g_{2}$.

If $K$ is abelian, $g_{1}=g_{2}$ and we one done. Suppose that $K$ is a nonabelian group having two generated free abelian subgroup of finite index. Choose $A$ a normal torsion free abelian subgroup of finite index, where 
without lost generality we can assume that $g_{1}^{m}$ is a generator of $A$. Let $\varphi: K \longrightarrow \operatorname{Aut}(A)=G L_{2}(\mathbb{Z})$ be the homomorphism induced by the action of $K$ on $A$. Since $N$ is central in $K, \varphi(K)$ is a finite subgroup of the group of the upper triangular matrices with first column $\left(\begin{array}{l}1 \\ 0\end{array}\right)$. Hence the other element of the diagonal of this matrix must be 1 ou -1 . Since the image of $K$ in $G L_{2}(\mathbb{Z})$ is finite and a matrix of the form $\left(\begin{array}{ll}1 & x \\ 0 & 1\end{array}\right)$ has infinite order, any element of $\varphi(K)$ has the form $\left(\begin{array}{cc}1 & x \\ 0 & -1\end{array}\right)$. But $\left(\begin{array}{cc}1 & x \\ 0 & -1\end{array}\right)\left(\begin{array}{cc}1 & y \\ 0 & -1\end{array}\right)=\left(\begin{array}{cc}1 & y-x \\ 0 & 1\end{array}\right)$, so we conclude that $\varphi(K)$ is a group of order 2 . Hence the centralizer $P=C_{K}(A)$ of $A$ in $K$ has index 2. Since $A \leq Z(P),[P, P]$ is finite and since $P$ is torsion free, $P$ is abelian. Therefore we can assume that $P=A$. In this case conjugating the image of $K$ in $G L_{2}(\mathbb{Z})$ if necessary (i.e., choosing another base for $A$ ) we can suppose that $\varphi(K)$ is generated either by the diagonal matrix $\left(\begin{array}{cc}1 & 0 \\ 0 & -1\end{array}\right)$ or by the matrix $\left(\begin{array}{cc}1 & 1 \\ 0 & -1\end{array}\right)$. Indeed, conjugating the matrix $\left(\begin{array}{cc}1 & x \\ 0 & -1\end{array}\right)$ by the matrix $\left(\begin{array}{cc}1 & y \\ 0 & -1\end{array}\right)$ we get $\left(\begin{array}{cc}1 & 2 y-x \\ 0 & -1\end{array}\right)$, then we get the first matrix in the case of $x$ being even, and the second matrix in the case of $y$ being odd; in the latter case the matrix is conjugate of the matrix $\left(\begin{array}{ll}0 & 1 \\ 1 & 0\end{array}\right)$.

In the first case $K$ is a generalized dihedral group. Indeed, let $z$ be an element of the inverse image of $\left(\begin{array}{cc}1 & 0 \\ 0 & -1\end{array}\right)$. Note that $Z(K)$ is cyclic, because otherwise $Z(K)$ is of finite index and since $K$ is torsion free, $K$ would be abelian. Therefore since $z$ centralizes $z^{2}, z^{2}$ is a power of $g_{1}^{m}$. But this power can be only 1 or -1 , otherwise factoring out $z^{2}$ we get that $K /\left\langle z^{2}\right\rangle$ has the followings generators $g_{1}^{m}\left\langle z^{2}\right\rangle, \alpha\left\langle z^{2}\right\rangle$ and $z\left\langle z^{2}\right\rangle$, where $\alpha$ is the generator of the group $A$ inverted by the matrix above; hence we have a 3 -generated group, unless $z^{2}$ is $g_{1}^{m}$ or $\left(g_{1}^{m}\right)^{-1}$. Thus $K$ has only one relation $\alpha^{z}=\alpha^{-1}$. Hence $K$ is a generalized dihedral group which is discarded by the hypothesis of the theorem.

In the second case, $K$ has torsion. Indeed, let $z$ be the inverse image of the $\left(\begin{array}{ll}0 & 1 \\ 1 & 0\end{array}\right)$, then there exist generators $x$ and $y$ of $A$ that are exchanged by the action of $z$, i.e., $x^{z}=y$ and $y^{z}=x$. Since $z$ centralizes $z^{2}, z^{2}=$ 
$(x y)^{n}$, for some $n \in \mathbb{Z}$. But $\left(z x^{-n}\right)^{2}=z x^{-n} z x^{-n}=z^{2}(y x)^{-n}=1$, i.e., $z x^{-n}$ has order 2 . Therefore $K$ has torsion and since $G$ is torsion free this case is also discarded.

Thus $K$ is isomorphic to $\mathbb{Z} \times \mathbb{Z}$, and this implies that $g_{1}=g_{2}$.

\section{Principal results}

Recall that the Bianchi Groups are $P S L_{2}\left(O_{d}\right)$, where $O_{d}$ is the ring of integers in the imaginary quadratic number field $\mathbb{Q}(\sqrt{-d})$, and

$$
O_{d}=\mathbb{Z}+w \mathbb{Z}, \text { where }\left\{\begin{array}{l}
w=\sqrt{-d}, \text { se }-d \equiv 1 \bmod (4) \\
w=\frac{1+\sqrt{-d}}{2}, \text { se }-d \not \equiv 1 \bmod (4) .
\end{array}\right.
$$

and $d$ is a positive square-free integer. Let $m \in \mathbb{N}$ and $O_{d, m}=\mathbb{Z}+m w \mathbb{Z}$, where $w$ is given above. It is easy to see that $\left|O_{d}: O_{d, m}\right|=m$, and we can consider the groups $S L_{2}\left(O_{d, m}\right)$. Observe that $S L_{2}\left(O_{d, m}\right)$ is of finite index in $S L_{2}\left(O_{d}\right)$.

We shall use the following decompositions of Bianchi groups $\Gamma_{d}=$ $P S L_{2}\left(O_{d}\right)$ for $d=1,2,7,11$ (see [F-89]).

$$
P S L_{2}\left(O_{1}\right)=G_{1} *_{M} G_{2},
$$

where $G_{1}=S_{3} *_{\mathbb{Z} / 3 \mathbb{Z}} A_{4}, G_{2}=S_{3} *_{\mathbb{Z} / 2 \mathbb{Z}} D_{2}$ and $M$ is the modular group $P S L_{2}(\mathbb{Z})$.

$$
\begin{aligned}
P S L_{2}\left(O_{2}\right) & =H N N\left(K_{2}, M, t\right) \\
P S L_{2}\left(O_{7}\right) & =H N N\left(K_{7}, M, t\right) \\
P S L_{2}\left(O_{11}\right) & =H N N\left(K_{11}, M, t\right),
\end{aligned}
$$

where $K_{2}=\left(A_{4} *_{\mathbb{Z} / 2 \mathbb{Z}} D_{2}\right), K_{7}=\left(S_{3} *_{\mathbb{Z} / 2 \mathbb{Z}} S_{3}\right), K_{11}=\left(A_{4} *_{\mathbb{Z} / 3 \mathbb{Z}} A_{4}\right)$ and $M$ is the modular group $P S L_{2}(\mathbb{Z})$.

The Bianchi group $\Gamma_{3}$ does not decompose in amalgamated free product or $H N N$-extension. Therefore we are going to use the decomposition found by Scarth of a certain subgroup of finite index in $\Gamma_{3}$. The decomposition is described in the next

Proposition 3.1 (Scarth, M. R.). There exists a subgroup $\Gamma_{3,2}:=P S L_{2}\left(O_{3,2}\right)$ of $\Gamma_{3}$ of finite index which is an $H N N$-extension of $K_{3,2}$ with the modular group associated, where $K_{3,2}=S_{3} *_{\mathbb{Z} / 3 \mathbb{Z}} D(3,3,3)$ and $D(3,3,3)=$ $\left\langle x, y \mid x^{3}=y^{3}=(x y)^{3}=1\right\rangle$. 
Proof. The group $P S L_{2}\left(O_{3,2}\right)$ has the following presentation (see [Sc-03]

$$
\left\langle a, t, w \mid a^{2}=(a t)^{3}=\left(w^{-1} a w a\right)^{3}=[t, w]=1\right\rangle .
$$

Let $v=w^{-1} a w$, then using Tietze transformations $\left((a v)^{3}=1\right.$ iff $(v a)^{3}=1$ and $(a t)^{3}=\left(w v w^{-1} t\right)^{3}=\left(w v t w^{-1}\right)^{3}=1$ iff $\left.(t v)^{3}=1\right)$ we get the following presentation

$$
\left\langle a, t, v, w \mid a^{2}=(a t)^{3}=(a v)^{3}=v^{2}=(t v)^{3}, t=w^{-1} t w, v=w^{-1} a w\right\rangle .
$$

Let $K_{3,2}=\left\langle a, t, v \mid a^{2}=(a t)^{3}=(a v)^{3}=v^{2}=(t v)^{3}\right\rangle$. We show that $P S L_{2}\left(O_{3,2}\right)$ is an $H N N$-extension of $K_{3,2}$ with $\langle a, t\rangle \cong M \cong\langle v, t\rangle$ associated.

Observe first that $\langle a, t\rangle \cong M$, since have the same presentation. Then $P S L_{2}\left(O_{3,2}\right)=H N N\left(K_{32}, M, w\right)$.

Let $s=a t, m=a v$. We get

$$
K_{3,2}=\left\langle a, s, m \mid a^{2}=s^{3}=m^{3}=(a m)^{2}=\left(s m^{2}\right)^{3}=1\right\rangle,
$$

where we used again that $s m^{2}=a t v a$ is of order 3 iff $t v$ is of order 3 . Observe that $\left\langle a, m \mid a^{2}=m^{3}=(a m)^{2}=1\right\rangle \cong S_{3}$ and a group with presentation $\left\langle s, \bar{m} \mid s^{3}=\bar{m}^{3}=\left(s \bar{m}^{-1}\right)^{3}\right\rangle \cong D(3,3,3)$. Then we have $K_{3,2}=S_{3} *_{m=\bar{m}} D(3,3,3)$.

Lemma 3.2. There exists a homomorphism $\tau: \Gamma_{3,2} \rightarrow P$, where $P$ is virtually a free product of torsion free abelian groups and a free group such that $\tau_{\mid K_{32}}$ is injective.

Proof. We use the notation of the preceding proposition. Let $\psi_{1}: S_{3} \rightarrow S_{3}$ be the map that sends $a \mapsto a m, m \mapsto m^{-1}$. Observe that $\left\{\psi_{1}(a), \psi_{1}(m)\right\}$ satisfies the relations of the group $S_{3}$, so $\psi_{1}$ is an epimorphism by Von Dick's theorem. Then $\psi_{1}$ is an automorphism of order 2 because am and $m^{-1}$ generate $S_{3}$. Let $\psi_{2}: D(3,3,3) \rightarrow D(3,3,3)$ be the map that sends $s \mapsto s^{-1}, \bar{m} \mapsto \bar{m}^{-1}$, again we have that $\psi_{2}$ is an automorphism of order 2. Note that these two automorphisms agree on the amalgamated subgroup $\langle m\rangle=\langle\bar{m}\rangle$, i.e., $\psi_{1}(m)=m^{-1}=\bar{m}^{-1}=\psi_{2}(\bar{m})$, therefore by the universal property of an amalgamated free product there exists a unique homomorphism $\psi: S_{3} *_{\mathbb{Z} / 3 \mathbb{Z}} D(3,3,3) \rightarrow S_{3} *_{\mathbb{Z} / 2 \mathbb{Z}} D(3,3,3)$ extending $\psi_{1}$ and $\psi_{2}$. Since $\psi_{1}$ and $\psi_{2}$ are automorphisms of order 2 it follows that $\psi$ is an automorphism of order 2 as well.

Consider the group $P=\left(S_{3} * \mathbb{Z} / 3 \mathbb{Z} D(3,3,3)\right) \rtimes\langle\psi\rangle$. Then the map $\varphi$ that sends $K_{3,2} \mapsto K_{3,2}$ identically and $w \mapsto \psi$, extends to an epimorphism $\tau$ : 
$\Gamma_{3,2} \rightarrow P$ by Von Dycks theorem because $\{\varphi(a), \varphi(m), \varphi(\bar{m}), \varphi(s), \varphi(w)\}$ satisfies the relations of $\Gamma_{3,2}$. In addition, $\left.\tau\right|_{K_{3,2}}$ is a monomorphism to $P$. Now it remains to note that by the Kurosh subgroup theorem (see [S-77] for the version with amalgamation) any torsion free subgroup of finite index of $P$ is a free product of virtually abelian group and a free group, since $D(3,3,3)$ is virtually free abelian.

Proposition 3.3. Let $H$ be finitely generated subgroup of $K_{32}$ and $M$ be the modular group. Then $\overline{H \cap M}=\bar{H} \cap \bar{M}$.

Proof. We must prove that $\overline{H \cap M} \geq \bar{H} \cap \bar{M}$, since, clearly $\overline{H \cap M} \leq$ $\bar{H} \cap \bar{M}$.

Consider $L=\left\langle K_{32}, K_{32}^{w^{-1}}\right\rangle=K_{32} *_{M} K_{32}^{w^{-1}}$ be a amalgamated free product of $K_{32}$, where $M$ is a modular group and set $P=\left\langle H, K_{32}^{w^{-1}}\right\rangle$. By the subgroup theorem for amalgamated free products we have $P=H *_{H \cap M}$ $K_{32}^{w^{-1}}$. Since $\Gamma_{32}$ is LERF, all the subgroups $H, M, P$ are closed in the profinite topology of $\Gamma_{32}$ and this topology induces an efficient profinite topology on $P$ and on $L$.

It follows that $\widehat{L}=\widehat{K_{32}} \amalg_{\widehat{M}}{\widehat{K_{32}}}^{w^{-1}}$ and $\bar{P}=\widehat{H} \amalg_{\widehat{H \cap M}} \widehat{K_{32}^{w^{-1}}}$.

By Exercise 9.2.7 (2) in [RZ] $\widehat{H} \amalg_{\widehat{H \cap M}} \widehat{K_{32}^{w^{-1}}}$ is proper. Obviously, $\widehat{H} \cap \widehat{K_{32}^{w^{-1}}}$ contains $\widehat{H} \cap \widehat{M}$. We show that $\widehat{H} \cap \widehat{K_{32}^{w^{-1}}}=\widehat{H \cap M}$. Indeed, suppose not. Pick an element $h \in \widehat{H} \cap \widehat{K_{32}^{w^{-1}}} \backslash \widehat{H \cap M}$. Then the exist an open normal subgroup $U$ of $\bar{P}$ such that $h U \notin \widehat{H \cap M} U / U$. Put $N=U \cap H, N^{\prime}=U \cap K_{32}^{w^{-1}}$ and $V=\widehat{H \cap M} \cap U$. Consider the natural homomorphism $f: P \longrightarrow H / N \amalg_{\widehat{H \cap M} / V} K_{32}^{w^{-1}} / N^{\prime}$. Then $f(h) \notin \widehat{H \cap M} / V$. But $f(h) \in f\left(\widehat{H} \cap \widehat{K_{32}^{w^{-1}}}\right) \subset(H / N) \cap\left(K_{32}^{w^{-1}} / N^{\prime}\right)=$ $\widehat{H \cap M} / V$. So $h \in K \operatorname{Ker}(f) \widehat{H \cap M} \leq \widehat{H \cap M} U$ contradicting with the fact that $h U \notin \widehat{H \cap M} U / U$. Thus $\widehat{H} \cap \widehat{K_{32}^{w-1}}=\widehat{H \cap M}$.

Proposition 3.4. Let $H$ be a finite index torsion free subgroup of $G=\Gamma_{d}$, $d=1,2,7,11$ or $G=\Gamma_{3,2}$. Then $H$ is conjugacy separable.

Proof. Let $S(G)$ and $S(\widehat{G})$ be the trees associated with decompositions of $G$ and $\widehat{G}$. By [L-R-2004, Theorem 3.6.1] $G$ is subgroup separable, therefore the profinite topology on $G$ is efficient, so $S(G)$ is embedded in $S(\widehat{G})$ (see Remark 2.77.

By Lemma 2.2 the centralizers of non-trivial elements of $H$ are free abelian groups of rank at most 2 . Hence by Theorem 2.17 it is enough to proof the proposition for an appropriate subgroup of finite index of $H$. An 
appropriate subgroup for us is a subgroup that satisfies the conditions of Lemma 2.16. We show the existence of such a subgroup. Indeed, by Lemmas 4.2 and 4.3 in [W-Z-98] and by Lemma 3.2 in the case $d=3$, there exists virtually a free product $P$ of torsion free abelian groups and a free group and an epimorphism $\tau: G \rightarrow P$ such that $\left.\tau\right|_{G_{v}}$ is injective, for all $v \in V(S)$ and $\left|P: \tau\left(G_{v}\right)\right|<\infty$. Therefore replacing $H$ by a subgroup of finite index if necessary we may assume that the image of $H$ is a free product of torsion free abelian groups and a free group. As $S(G) / H$ is finite, there exists a finite number of orbits $H v, v \in S(G)$, i.e. there exists a finite number of vertex stabilizers in $H$ up to conjugation. Since the index $\left[P: \tau\left(G_{v}\right)\right]$ is finite, so is $\left[P: \tau\left(H_{v}\right)\right]$. It follows that $\tau\left(H_{v}\right)$ has a finite number of conjugates in $P$. Therefore $K:=\bigcap_{v \in V(S)} \tau\left(H_{v}\right)$ has finite index in $P$ and $L=\tau^{-1}(K) \cap H$ is the desired subgroup. Indeed, $\left.\tau\right|_{L_{v}}$ is an isomorphism to $K$, because $\tau\left(L_{v}\right)=\tau\left(L \cap H_{v}\right)=\tau(L)$, where the last equality follows from the definition of $L$ (to see that the right handside is contained in the left handside let $l \in L$; then $\tau(l) \in K \leq \tau\left(H_{v}\right)$; hence there exist $h_{v} \in H_{v}$, $x \in \operatorname{Ker}(\tau)$ such that $l=x h_{v}$; but $\operatorname{Ker}(\tau) \leq \tau^{-1}(K)$, so $\left.h_{v} \in L\right)$. Moreover, the vertex and edges stabilizers in $L$ are finitely generated and since $G$ is subgroup separable [L-R-2004, Theorem 3.6.1], the profinite topology on $G$ induces the efficient profinite topology on $L$. Note also that every finitely generated subgroup of $K$ is conjugacy distinguished by Proposition 2.13, because $K$ is isomorphic to a subgroup of $P$ and so by the Kurosh subgroup theorem satisfy the hypothesis of Proposition 2.13. Thus replacing $H$ by $L$ if necessary, from now on we may assume that $H$ satisfies the hypotheses of Lemma 2.16.

Let $g_{1}, g_{2} \in H$ such that $g_{2}=\gamma^{-1} g_{1} \gamma$ for any $\gamma \in \widehat{H}$. We show that $g_{1}$ and $g_{2}$ are conjugate in $H$. We split the proof into two cases.

Case 1: $g_{1}$ is non-hyperbolic. By item (i) of Proposition 2.9 in [R-Z-96] $g_{2}$ is non-hyperbolic as well. Let $v_{1}$ be a vertex fixed by $g_{1}$. If $\gamma \in \widehat{G}_{v_{1}}$, then $g_{2}=g_{1}^{\gamma} \in \widehat{G}_{v_{1}}$. Since $\widehat{G}_{v_{1}} \cap H=G_{v_{1}} \cap H$, the elements $g_{1}, g_{2} \in G_{v_{1}} \cap H$. By Lemma $2.5 \widehat{G}_{v_{1}} \cap \widehat{H}=\widehat{G_{v_{1}} \cap H}$, so $\gamma \in \widehat{G_{v_{1}} \cap H}$. Since $H$ satisfies the hypothesis of Lemma 2.16 the group $H_{v_{1}}=G_{v_{1}} \cap H$ satisfies hypothesis of Proposition 2.13 and so it is conjugacy separable. Thus there exists $\gamma_{1} \in$ $G_{v_{1}} \cap H$ such that $g_{2}=g_{1}^{\gamma_{1}}$, i.e. $g_{1}$ and $g_{2}$ are conjugate in $H \cap G_{v_{1}}$.

Suppose now that $\gamma \notin \widehat{G}_{v_{1}}$. We shall prove then that some conjugates of $g_{1}$ and $g_{2}$ in $H$ both stabilize some edge $e \in S$.

By Theorem 3.12 [Z-M-89] $g_{1}=\gamma g_{2} \gamma^{-1} \in \widehat{G}_{v_{1}} \cap \gamma \widehat{G}_{v} \gamma^{-1} \leq \widehat{G}_{\bar{e}}$, where $\bar{e} \in S(\widehat{G}), v$ is a vertex of $S(G)$ stabilized by $g_{2}$ and $v=d_{0}(\bar{e})$ or $v=d_{1}(\bar{e})$. Now $\left|G_{v}: H_{v}\right|<\infty$, so $\widehat{G}_{v} / \widehat{H}_{v}=G_{v} / H_{v}$ and we 
get $\operatorname{Star}_{S(\widehat{G})}(v) / \widehat{H}_{v}=\operatorname{Star}_{S(G)}(v) / H_{v}$. This implies the existence of an element $\widehat{h} \in \widehat{H_{v}}$ such that $e_{1}:=\widehat{h} \bar{e} \in S(G)$.

Then $g_{1}^{-1} \in \widehat{H_{e_{1}}}$ and since $\widehat{h} \in \widehat{H_{v}}$, and $H_{e_{1}}$ is conjugacy distinguished in $H_{v}$ (by item 5 of Proposition 2.13), there exists $h \in H_{v}$ such that $g_{1}^{h} \in$ $H_{e_{1}}$.

Thus we may assume that $g_{1} \in H_{e}$, where $e$ is an edge of $S(G)$. By Lemma 2.16 the edge stabilizers $H_{e}$ are conjugacy distinguished in $H$. Therefore conjugating $g_{2}$ by an element of $H$ if necessary we may assume that $g_{2} \in H_{e}$.

Recall now that $\tau: H \longrightarrow K$ is a homomorphism satisfying hypothesis of Lemma2.16 As $g_{1}, g_{2}$ are conjugate in $\widehat{H}$, the elements $\tau\left(g_{1}\right)$ and $\tau\left(g_{2}\right)$ are conjugate in $\widehat{K}$, and since $K$ is conjugacy separable there exists $k \in K$ such that $\tau\left(g_{2}\right)=\tau\left(g_{1}\right)^{k}$. Then there exists $z \in H \cap G_{v_{1}}$ such that $\tau(z)=k$, so $\tau\left(g_{2}\right)=\tau\left(g_{1}^{z}\right)$, and since $g_{2}, g_{1}^{z} \in G_{v_{1}} \cap H$ and $\left.\tau\right|_{G_{v_{1}}}$ is injective, one has $g_{2}=g_{1}^{z}$.

Case 2: The elements $g_{1}, g_{2}$ are hyperbolic. Let $T_{g_{1}}$ and $T_{g_{2}}$ be the infinite straight lines on which $g_{1}$ and $g_{2}$ act. Let $e$ be any edge of $T_{g_{1}}$. Since $g_{2}=\gamma^{-1} g_{1} \gamma$, the group $\left\langle g_{2}\right\rangle$ acts freely over $\gamma^{-1} \overline{T_{g_{1}}}$, and by Proposition 2.2(ii) in [R-Z-96] $\gamma^{-1} \bar{T}_{g_{1}}=\bar{T}_{g_{2}}$. It follows that $\gamma^{-1} e \in \bar{T}_{g_{2}}=\overline{\left\langle g_{2}\right\rangle} T_{2}$, where $T_{2}=\left[v_{2}, g_{2} v_{2}\right]$ for any $v_{2} \in T_{g_{2}}$. Let $x \in \overline{\left\langle g_{2}\right\rangle}$ such that $x \gamma^{-1} e \in T_{2}$ and $\widehat{\mu}: S(\widehat{G}) \rightarrow S(\widehat{G}) / \widehat{H}$ be the natural map. Then $\widehat{\mu}\left(x \gamma^{-1} e\right)=\widehat{\mu}(e)$, and since $S(\widehat{G}) / \widehat{H}=S(G) / H$ (as one easily checks using the closedness of $H G_{e}$ in $G$ ) there exists $g \in H$ such that $x \gamma^{-1} e=g e \in S$. Therefore $g^{-1} x \gamma^{-1} e=e$ and so $\delta:=g^{-1} x \gamma^{-1} \in \overline{H_{e}}$. Note that $g_{1}=\gamma g_{2} \gamma^{-1}=$ $\gamma x^{-1} g_{2} x \gamma^{-1}=\delta^{-1} g^{-1} g_{2} g \delta$. Then substituting $g_{2}$ with $g^{-1} g_{2} g$ and $\gamma$ with $\delta$ we can suppose that $\gamma \in \overline{H_{e}}$ and so $T_{g_{1}}$ and $T_{g_{2}}$ have a common edge $e$.

Let $P$ be a geodesic of finite length in $T_{g_{1}}$ that has $e$ as one of its edges and such that $\gamma \in \bar{I}$, where $I=\underset{e \in E(P)}{\bigcap} H_{e}$. We show that we can assume that $\gamma$ can be substituted by an element that belongs to the closure of intersection of the edge stabilizers of a geodesic which strictly contains $P$. Let $e_{1} \in$ $T_{g_{1}} \backslash P$ be an edge connected to $P$, and let $v$ be the common vertex of $e_{1}$ and $P$. Let $P^{+}=P \cup\left\{e_{1}\right\}$, and put $e_{2}=\gamma^{-1} e_{1}$ so that $e_{2} \in \bar{T}_{g_{2}}$, because $\gamma^{-1} \bar{T}_{g_{1}}=\bar{T}_{g_{2}}$. In fact, $e_{2} \in T_{g_{2}}$. Indeed, $\gamma^{-1}\left[e, e_{1}\right]=\left[e, e_{2}\right]$ is finite and contained in $\overline{T_{g_{2}}}$. Now observe that $T_{g_{2}}$ is a connected component of $\bar{T}_{g_{2}}$ considered as an abstract graph, in others words the only vertices of $\bar{T}_{g_{2}}$ that are at a finite distance from $T_{g_{2}}$ are the vertices of $T_{g_{2}}$, see Proposition 2.10 (iv). Therefore $e_{2} \in T_{2}$.

Let $\mu: S(G) \longrightarrow S(G) / H$ be the natural map. Since $S(G) / H=$ 
$S(\widehat{G}) / \widehat{H}$ we have $\mu\left(e_{1}\right)=\widehat{\mu}\left(e_{1}\right)=\widehat{\mu}\left(e_{2}\right)=\mu\left(e_{2}\right)$, so there exists $h \in H$ such that $e_{1}=h e_{2}$. Since $e_{1}$ and $e_{2}$ have a common vertex $v$, we have $h \in H_{v}$. Therefore, $e_{2}=h^{-1} e_{1}=h^{-1} \gamma e_{2}$ and so $\gamma_{1}:=h^{-1} \gamma \in \overline{H_{e_{2}}}$.

Recall that stabilizers of vertices in $H$ are finitely generated and so by Theorem 1 in [B-C-K-98], $I$ is finitely generated. Since $G_{v}$ is subgroup separable (Proposition 2.13), the groups $I$ and $H_{e_{2}}$ are closed in the profinite topology of $G_{v}$. Therefore by the main result in [C-2001] $I H_{e_{2}}$ is closed in the profinite topology of $G_{v}$, i.e., $\overline{I H_{e_{2}}} \cap G_{v}=I H_{e_{2}}$. Thus $h=\gamma \gamma_{1}^{-1} \in$ $I H_{e_{2}}$ and so there exist $h_{1} \in I, h_{2} \in H_{e_{2}}$ such that $h=h_{1} h_{2}$. Let $\gamma^{+}=$ $\gamma h_{1}^{-1}$, then $\gamma^{+} e_{1}=\gamma h_{1}^{-1} e_{1}=\gamma h_{2} h^{-1} e_{1}=\gamma e_{2}=e_{1}$, and therefore $\gamma^{+} \in \bar{H}_{e_{1}}$. We also have $\gamma^{+}=\gamma h_{1}^{-1} \in \bar{I}$, and since $I$ and $H_{e_{1}}$ are finitely generated in $H_{v}$, by Proposition 2.4 in [W-Z-98] for the cases $d=1,2,7,11$ and by Lemma 3.3 for the case $d=3$ we have $\gamma^{+} \in \overline{\cap_{e \in P^{+}} H_{e}}$. Therefore replacing $\gamma$ with $\gamma^{+}$and $g_{2}$ with $h_{1} g_{2} h_{1}^{-1}$ we may assume that $\gamma \in \overline{\underset{e \in P^{+}}{\cap} H_{e}}$.

Thus we always can assume that $P$ has sufficiently large length. Let $P$ be a geodesic of $T_{g_{1}}$ that contains $e$ and $g_{1} e$. Let $I=\bigcap_{e \in P} H_{e}$ and consider $D=I \cap g_{1} I g_{1}^{-1}$. Note that $g_{1} I g_{1}^{-1}$ is the intersection of the stabilizers of the path $g_{1} P$. Now by what was done above, we can suppose that $\gamma \in \bar{D}$ (we use $P^{+}=P \cup g_{1} P$ and if $e^{\prime}=g_{1} e$, then $H_{e^{\prime}}=g_{1} H_{e} g_{1}^{-1}$ ). Observe that $I$ is cyclic, because the intersection of two edge groups in $S(G)$ is cyclic, by Lemma 4.1 in [W-Z-98]. Now we prove that $g_{1}$ normalizes $D$. Indeed, since $D$ is closed in $G$, then $D=\cap_{N \unlhd_{f} G} D N$. Thus it is enough to proof that $D N$ is normalized by $g_{1}$, for each $N \unlhd_{f} G$. Indeed, let $N \unlhd_{f} G$ and consider the quotient $G / N$. Then the group $D N / N$ have the same index $m$ in the groups $I N / N$ and $I^{g_{1}^{-1}} N / N$, because these groups are conjugate. If $x N$ generates $I N / N$, then $(x N)^{m}$ and $\left(g_{1} N\right)(x N)^{m}\left(g_{1} N\right)^{-1}$ generate $D N / N$ and we conclude that $D N$ is normalized by $g_{1}$.

Let $d$ be a generator of $D$ and write $E=\left\langle d, g_{1}\right\rangle$. We proof that $E$ is abelian. Indeed, if $d$ does not centralize $g_{1}, g_{1} d g_{1}^{-1}=d^{-1}$ and so $E$ is a generalized dihedral group. However, by Corollary 2.3 the Bianchi groups do not contain subgroups isomorphic to the generalized dihedral group, a contradiction.

Therefore $d$ centralizes $g_{1}$ and consequently $E$ is abelian. This implies that $\bar{E}$ is abelian and so $g_{1}=g_{2}$, as needed.

Theorem 3.5. Let $G$ be a torsion free group commensurable with a Euclidean Bianchi group. Suppose that $G$ does not contain subgroups isomorphic to the generalized dihedral group. Then $G$ is conjugacy separable. 
Proof. Since $G$ is commensurable with $\Gamma_{d}$ (note that commensurability with $\Gamma_{3}$ is equivalent to commensurability with $\Gamma_{3,2}$ ), there exist isomorphic subgroups $M, N$ of finte index in $G$ and $\Gamma_{d}$ respectively. Then by the preceding proposition $N$ is conjugacy separable. Consequently $M$ is conjugacy separable. Then $M \leq G$ satisfies all hypothesis of Theorem 2.17, hence $G$ is conjugacy separable.

Theorem 3.6. Let $G \leq P G L_{2}(\mathbb{C})$ be a torsion free group commensurable with $\Gamma_{d}, d=1,2,3,7,11$. Then $G$ is conjugacy separable.

Proof. Follows from Theorem 3.5 and Corollary 2.3 .

Corollary 3.7. The figure eight knot group is conjugacy separable.

Proof. The figure eight knot group is a torsion free subgroup of index 12 in $\Gamma_{3}=P S L_{2}\left(O_{3}\right)$ (see page 60 in [M-R-02]), so by Theorem $3.6 \mathrm{~K}$ is conjugacy separable.

Remark 3.8. In Theorem $3.6 P G L_{2}(\mathbb{C})$ can be replaced by $G L_{2}(\mathbb{C})$ because a subgroup of $G L_{2}(\mathbb{C})$ containing the generalized dihedral group $\left\langle x, y \mid x^{2}=y^{2}\right\rangle$ must have $x^{2} \in Z\left(G L_{2}(\mathbb{C})\right.$ by Lemma 2.1 and so can not be commensurable with $\Gamma_{d}$. Indeed, every subgroup of finite index in $\Gamma_{d}$ has finite center.

\section{References}

[B-71] Burns, R. G., On Finitely Generated Subgroups of Free Products, J. Austral. Math. Soc., 12 (1971) 358-364.

[B-C-K-98] Burns R. G., Chau E. C., Kam S.-M., On The Rank of Intersection of Subgroups of a Free Product of Groups, J. Pure Appl. Algebra, 124 (1998), 31-45.

[C-2001] Coulbois T., Free Product, Profinite Topology and Partial Action of Groups on Relational Structures: A Connection Between Model Theory and Profinite Topology, World Scientific, Singapore 2002, 349361.

[D-80] Dicks W., Groups, Trees and Projective Modules, Lecture Notes in Mathematics, No. 790, Springer-Verlag, 1980.

[D-D-89] Dicks W., Dunwoody M. J., Groups Acting on Graphs, Cambrige Univ. Press., Cambrige, 1989. 
[Dy-79] Dyer J. L., Separating Conjugates in Amalgamated Free by Finite Groups, J. London Math. Soc., 2, 20 (1979) 215-222.

[F-89] Fine B., Algebraic Theory of the Bianchi Groups, Marcel Dekker, 1989.

[G-M-S-2003] Gitik R., Margolis S.W., Steinberg B., On the Kurosh theorem and separability properties, Journal of Pure and Applied Algebra, 179 (2003) 87-97.

[H-R-85] Herfort W. H., Ribes L., Torsion Elements and Centralizers in Free Products of Profinite Groups, J. Reine Angew. Math., 358 (1985) 155-161.

[L-R-2004] Long D. D., Reid A. W., Subgroup Separability and Virtual Retractions of Groups, Preprint, 23pp.

[M-58] Mal'cev A. I., On Homormorphisms onto Finite Groups, Uchen. Zap. Ivanovskogo Gos. Ped. Inst., 18 (1958) 40-60.

[M-R-02] Maclachlan C., Reid A. W., The Arithmetic of Hyperbolic 3Manifolds, Graduate Texts in Mathematics, 219, Springer, 2002.

[N-92] Niblo G. A., Separability Properties of Free Groups and Surface Groups, J. Pure Appl. Algebra, 78 (1992) 77-84.

[R-71] Remeslennikov V. N., Groups that are Residually Finite with Respect to Conjugacy, Siberian Math. J., 12 (1971) 783-792.

[Ro-69] Romanovskii V. N., On the Residual Finiteness of Free Products with Respect to Subgroups, Izv. Akad. Nauk SSSR Ser. Mat., 33 (1969) 1324-1329.

[R-T-V-95] Raptis E., Talelli O., Varsos D., On the Conjugacy Separability of Certain Graphs of Groups, J. Algebra, 199 (1998) 327-336.

[R-S-Z-98] Ribes L., Segal D., Zalesskii P. A., Conjugacy Separability And Free Products of Groups with Cyclic Amalgamation, J. London Math. Soc., 2, 57 (1998) 609-628.

[RZ] L. Ribes, P. Zalesskii, Profinite groups. Ergebnisse der Mathematik und ihrer Grenzgebiete, 3. Folge 40, Springer-Verlag, Berlin, (2000).

[R-Z-96] Ribes L., Zalesskii P. A., Conjugacy Separability of Almagamated Free Products of Groups, Journal of Algebra, 179 (1996) 751-774.

[R-Z-93] Ribes L., Zalesskii P. A., On the Profinite Topology of a Free Group, Bull. London Math. Soc., 25 (1993) 37-43. 
[R-Z-04] Ribes L., Zalesskii P.A., Profinite topologies in Free Products of Groups. International Conference on Semigroups and Groups in honor of the 65th birthday of Prof. John Rhodes. Internat. J. Algebra Comput. 14 (2004), no. 5-6, 751-772.

[S-70] Serre J.P., Le Probleme de Groupes de Congruence pour $S L_{2}$, Ann. of Math., 92 (1970) 489-657.

[S-77] Serre J.P., Trees, Springer Monographs in Mathematics, Springer, 1977.

[Sc-03] Scarth M. R., PhD Thesis: Normal Congruence Subgroups of Bianchi Groups and Related Groups, Faculty of Science, University of Glasgow (2003).

[W-Z-98] Wilson J. S., Zalesskii P. A., Conjugacy Separability of Certain Bianchi Groups and HNN Extensinos, Math. Proc. Camb. Phil. Soc., 123 (1998) 227-242.

[Z-M-89] Zalesskii P. A., Mel'nikov O. V., Subgroups of Profinite Groups Acting on Trees, Math. Sb., 63 (1989) 405-424.

[Z-M-90] Zalesskii P. A., Mel'nikov O. V., Fundamental Groups of Graphs of Profinite Groups, Algebra i Analiz, 1 (1989) 117-134.

[Z-T-95] Zalesskii P. A., Tavgen O. I., Closed Orbits and Finite Approximability With Respect to Conjugacy of Free Almagamated Products, Mathematical Notes, 58 (1995) 1042-1048.

[Z-92] Zalesskii P. A., Open Subgroups of Free Profinite Products, Contemporary Mathematics, 131 (1992) 473-491.

[Z-89] Zalesskii P. A., A Geometrical Characterization of Free Constructions of Profinite Groups, Siber. Math. J., 30 (1989) 73-84.

Department of Mathematics

University of Amazonas

Avenida General Rodrigo

Octávio Jordão Ramos, 3000

Aleixo 69000-077

Manaus-AM

Brazil

Department of Mathematics

University of Brasília

Brasilia-DF 70910-900

Brazil

pz@mat.unb.br

sheilachagas@ufam.edu.br 